\title{
ORIGINAL
}

\section{PREVENCIÓN SECUNDARIA DE CARDIOPATIA ISQUÉMICA A NIVEL LIPÍDICO EN ATENCIÓN PRIMARIA ARAGON. ESTUDIO PRECIAR 1}

\author{
A.A. Martínez Hernández (1), M.J. Aguilar Leñero (1), M. Rabadán Mengíbar (1), F. Hernansanz \\ Iglesias (2), J. González Ramos (3) y A. Marín Obáñez (3) \\ (1) Centro de Salud Fuente Norte. Zaragoza. \\ (2) Centro de Salud San José Norte. Zaragoza. \\ (3) Grupo GIMZ Zaragoza

\section{RESUMEN} \\ Fundamento: A pesar de la evidencia existente sobre la \\ eficacia de la prevención secundaria, modificando los estilos de \\ vida o utilizando los diversos fármacos que han mostrado su \\ beneficio clínico en pacientes coronarios, la información dis- \\ ponible sobre el manejo terapéutico de estos pacientes en el \\ ámbito de la atención primaria en nuestro país es escasa y poco \\ ABSTRACT

\section{Secondary Prevention of Ischemic Heart Disease at the Lipid-Related Level in Primary Care in Aragon. Study PRECIAR I} precisa. El objetivo de este estudio es conocer el estado de la prevención secundaria de la enfermedad coronaria desde el punto de vista del control de los lípidos.

Métodos: Se revisaron las historias clínicas de todas las personas diagnosticadas en algún momento de su vida de patología cardiovascular isquémica; incluyéndose en este apartado el infarto agudo de miocardio y el ángor, dentro de 11 cupos de 3 Centros de Salud urbanos del área II de Zaragoza. La población global era de 19.692 pacientes, encontrándose 388 casos de cardiopatía isquémica, de los datos recogidos de las historias.

Resultados: Estudiando los datos sobre el control lipídico, el $60.8 \%$ de los casos analizados en este estudio presentaba un perfil lipídico completo en el último año. De estos 236 pacientes solamente el $4,7 \%$ tenía el $\mathrm{LDL}<100$, teniendo que limitar a un c-LDL $<130$ el objetivo de control para encontrar un $31,3 \%$ de pacientes con un control subóptimo de este parámetro.

Conclusiones: Los resultados de nuestro estudio revelan que el diagnóstico de las dislipemias, así como el control del resto de factores de riesgo cardiovascular en prevención secundaria no se han adecuado en gran medida a los criterios de calidad de este proceso asistencial y señalan, en consecuencia, la existencia de un amplio margen de mejora.

Palabras clave: Cardiopatía isquémica. Prevención secundaria. Perfil lipídico. Atención primaria de salud.

Correspondencia:

Centro de salud Fuentes Norte

Dr Iranzo s/n

50.002 Zaragoza

Correo electrónico: adrimh@yahoo.es
Background: Despite the existing evidence of the effectiveness of secondary prevention by modifying lifestyles or using the different drugs which have shown themselves to be clinically beneficial for heart patients, there is little, not highly accurate information available regarding the handling of the treatment of these patients within the scope of primary care in our country. The purpose of this study is that of ascertaining the current status of secondary prevention of heart disease as far as lipid control is concerned.

Methods: A review was made of the clinical records of all of the patients diagnosed at some point in time of their life as having ischemic heart disease, including those under the heading of acute myocardial infarction and angina pectoris within the 11 groups of patients assigned to three urban Health Care Centers in Area II in Zaragoza. The population in question totaled 19,692 patients, 388 cases of ischemic heart disease having been found in the record files data.

Results: Based on a study of the lipid control data, $60.8 \%$ of the cases analyzed in this study showed a complete lipid profile for the last year. Solely $4.7 \%$ of these patients had LDL < 100 , the control objective having to be limited to $\mathrm{c}-\mathrm{LDL}<130$ to find a $31.3 \%$ of patients with a lower than optimum control of this parameter.

Conclusions: The results of our study reveal that the diagnosis of dyslipemias as well as the control of all other heart disease risk factors in secondary care have not been in keeping, for the most part, with the quality criteria of this health care procedure and therefore reveal the existence of a major degree of room for improvement.

Keywords: Ischemic heart disease. Secondary prevention. Lipid profile. Primary care. 


\section{INTRODUCCIÓN}

La asociación entre hipercolesterolemia y arterioesclerosis está bien establecida, existiendo evidencias concluyentes de causalidad, tanto desde el punto de vista clínico como anatomopatológico. Como ya demostró el estudio MRFIT, esta asociación se manifiesta, fundamentalmente con un riesgo gradual y continuo de presentar un episodio coronario, que aumenta progresivamente a medida que se incrementan las cifras de CT sin que se haya podido delimitar cual es el dintel a partir del cual aparece el incremento del riesgo.

La evidencia del beneficio de las intervenciones farmacológicas con fármacos hipolipemiantes es concluyente, a pesar de que no en todos los estudios se llegue a demostrar; de hecho, éste ya era conocido incluso antes de la aparición de los inhibidores de la hidroximetilglutaril coenzima A reductasa (estatinas). Cabe destacar, sin embargo, por todo lo que han contribuido para mejorar el tratamiento de los pacientes con enfermedad coronaria, los estudios realizados con estatinas: el Scandinavian Simvastatin Survival Study (4S) ${ }^{5}$, el Cholesterol and Recurrent Events (CARE) ${ }^{4}$, y el Long-term Intervention with Pravastatin in Ischemic Disease (LIPID) ${ }^{1}$. Estos estudios demostraron una disminución significativa de la morbimortalidad coronaria. La mortalidad coronaria, dependiendo del estudio, disminuyó del 24 al $42 \%$ y también lo hizo la mortalidad total (del 22 al 30\%) ) $^{10-12}$. A pesar de la evidencia existente sobre la eficacia de la prevención secundaria, modificando los estilos de vida o utilizando los diversos fármacos que han mostrado su beneficio clínico en pacientes coronarios, la información disponible sobre el manejo terapéutico de estos pacientes en el ámbito de la atención primaria en nuestro país es escasa y poco precisa $^{3}$. Refiriéndonos al estudio $4 \mathrm{~S}$, y habiéndose demostrado el beneficio clínico del tratamiento hipolipemiante en la prevención secundaria de la cardiopatía coronaria, sería interesante conocer, tal y como hizo el estudio CARE aunque con pocos pacientes, si tras tratamiento prolongado con un fármaco hipolipemiante en pacientes de cardiopatía isquémica y concentraciones de colesterol no elevadas, se disminuye el riesgo de aparición de episodios recurrentes de isquemia.

Los objetivos del estudio son conocer el estado de la prevención secundaria de la enfermedad coronaria, en cuanto a control de lípidos se refiere, en las consultas de Atención Primaria del área II de la ciudad de Zaragoza. Para ello se determinó:

Porcentaje de pacientes que alcanzan el objetivo de c-LDL $<100 \mathrm{mg} / \mathrm{dl}$ establecido para pacientes en prevención secundaria ${ }^{16}$.

Porcentaje de pacientes en prevención secundaria, que presentan una correcta cumplimentación en su historia clínica de los siguientes parámetros: antropometría, ECG, hábito tabáquico, perfil lipídico completo (PLC).

Porcentaje de pacientes en prevención secundaria que siguen algún tipo de terapia farmacológica hipolipemiante, en función de sus niveles de colesterol LDL.

\section{MATERIAL Y MÉTODOS}

Se trata de un estudio descriptivo transversal realizado durante los meses de octubre y noviembre de 1999. Se revisaron las historias clínicas de todos las personas diagnosticadas de patología cardiovascular isquémica, incluyéndose en este apartado el infarto agudo de miocardio y el ángor según la clasificación CIE-10; la población estudiada fue la correspondiente a 11 cupos de tres Centros de Salud urbanos del área II de Zaragoza. La población global eran 19.692 personas, encontrándose 388 casos de cardiopatía isquémica, de los datos recogidos de las historias.

Rev Esp Salud Pública 2001, Vol. 75, N. ${ }^{\circ} 2$ 
Como variables de estudio se analizó la presencia o no en la historia clínica de los datos antropométricos (peso y talla), hábito tabáquico (si consta o no), ECG en el último año, glucemia, perfil lipídico, toma tensional en el último año y si seguían tratamiento hipolipemiante y antihipertensivo.

Consideramos perfil lipídico completo cuando al paciente se le había solicitado al menos colesterol total, triglicéridos y ambas fracciones del colesterol, c-HDL y c-LDL. Aceptamos como criterio óptimo de control los niveles de LDL-colesterol $<100 \mathrm{mg} / \mathrm{dl}$, tensión arterial sistólica (TAS) $<140 \mathrm{mmHg}$ y tensión arterial diastólica $(\mathrm{TAD})<90$ $\mathrm{mmHg}$; a su vez consideramos niveles subóptimos de control un LDL-colesterol $<130$ $\mathrm{mg} / \mathrm{dl}$, TAS $<160 \mathrm{mmHg}$ y $\mathrm{TAD}<100$ mmHg, según los criterios de la NCEP-II y del sexto comité para la prevención y tratamiento de la hipertensión ${ }^{15,16}$.

Se consideraron pacientes mal controlados a todos los sujetos con cifras superiores a las indicadas en el control subóptimo y a todos aquéllos a los que no se les había realizado alguna de las determinaciones analíticas durante el año anterior al estudio.

\section{RESULTADOS}

Respecto a la cumplimentación de los datos recogidos en las historias clínicas de los sujetos con patología cardiovascular isqué- mica, constaban los datos antropométricos en el $76 \%$ de los casos; tenían ECG realizado en el último año el 71,1\%; presentaban un perfil lipídico completo en el $60,8 \%$ de los casos; constaba el hábito tabáquico en el $77,1 \%$; la glucemia en el último año apareció en el $86,6 \%$ y el $91 \%$ casos presentaban una toma tensional en el último año (tabla 1).

La población venía definida por una edad media de 72,4 años $(+/-9,5)$, con un ligero predominio $(57,2 \%)$ de los varones. Calculamos un colesterol medio 221,78 (+/$40,2)$, siendo la media de sus fracciones $50,63(+/-14,2)$ para el HDL y 149,72 $(+/-34,1)$ para el LDL.

Estudiando los datos sobre el control lipídico, el $60,8 \%$ de los casos analizados en el presente estudio presentaba un perfil lipídico completo en el último año. De estas 236 personas solamente el 4,7\% tenia el LDL < 100 , teniendo que limitar a un c-LDL $<130$ el objetivo de control para encontrar un $31.3 \%$ de pacientes con un control subóptimo de este parámetro (tabla 2).

Se incluyen los porcentajes de consecución de objetivos de control de c-LDL, tanto en el conjunto de los 236 pacientes con perfil lipídico completo disponible, como en el subgrupo de 102 pacientes con PLC en tratamiento hipolipemiante.

Tabla 1

Tabla de cumplimentación de datos Historia clínica. $\mathbf{N}=388$

\begin{tabular}{|lcc|}
\hline \multicolumn{1}{|c}{ Cumplimentación Historia clínica } & $N$ & Porcentaje \\
\hline Antropometría & 295 & 76 \\
ECG & 276 & 71,1 \\
Habito tabáquico & 299 & 77,1 \\
Glucemia último año & 336 & 86,6 \\
Perfil lipídico completo & 236 & 60,8 \\
Toma tensional & 364 & 93,8 \\
Tratamiento hipolipemiante & 125 & 32,2 \\
Tratamiento antihipertensivo & 268 & 69 \\
\hline
\end{tabular}


Tabla 2

Tabla comparativa de valores LDL-colesterol

\begin{tabular}{|ccc|}
\hline Valores LDL & Global & En tto hipolipem. \\
\hline$<100$ & $4.7 \%$ & $4 \%$ \\
$<130$ & $31.3 \%$ & $24.2 \%$ \\
$>130$ & $64 \%$ & $71.8 \%$ \\
& 236 casos & 102 pacientes \\
\hline
\end{tabular}

Figura 1

Algoritmo del Estudio PRECIAR

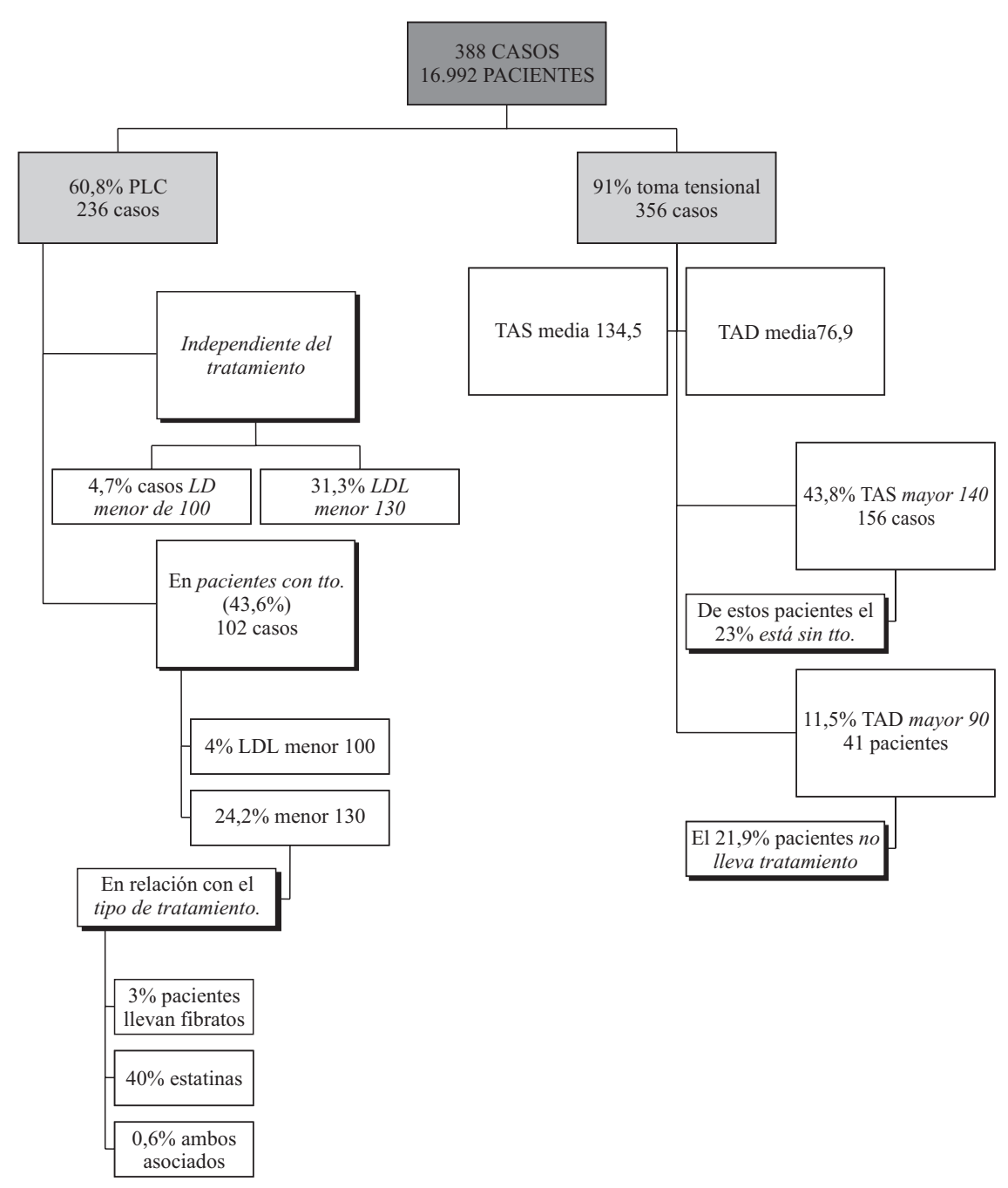


Relacionando el tratamiento hipolipemiante con las cifras de CT y LDL, hemos observado que alrededor del $56,6 \%$ de los 236 pacientes con un PLC no llevaban tratamiento hipolipemiante, el 3\% utilizaba fibratos, el $40 \%$ estatinas y el $0,4 \%$ ambos fármacos asociados. De estos 102 pacientes en tratamiento, únicamente el 4\% mantenía niveles de LDL por debajo de 100 y un $24,2 \%$ por debajo de 130 , variando el control medio del CT y sus fracciones según el tratamiento con dieta, fibratos, estatinas o asociadas.

Aunque no estaba incluido en nuestros objetivos, es interesante destacar que de los 356 pacientes con una toma tensional en el último año se observa una TAS media 134,53 (DE 17,33) y TAD media 76,99 (DE $9,21)$. De estos 364 pacientes el $43,8 \%$ presentan TAS 140 , y el $11,5 \%$ tienen TAD

90. De los 156 casos con mal control de la TAS el $23 \%$ estaba sin tratamiento médico. El 21,9\% de los 41 pacientes con TAD elevada se encontraban sin tratamiento (tabla 3 ).

\section{DISCUSIÓN}

Hasta la aparición de las estatinas, los estudios de intervención con fármacos hipolipemiantes presentaban resultados pobres por lo que respecta a la reducción de morbimortalidad cardiovascular y mortalidad total ${ }^{1-5}$. El estudio $4 \mathrm{~S}$ demostró que la reducción media del c-LDL conseguida tras 5,4 años de tratamiento fue del $35 \%$, conseguida con simvastatina en pacientes de prevención secundaria con cifras elevadas de colesterol total (entre 212 y $309 \mathrm{mg} / \mathrm{dl}$ ) era capaz de acompañarse de un importante descenso de morbimortalidad cardiovascular, tan importante que, al no modificarse la mortalidad no cardiovascular, se producía un descenso significativo de la mortalidad total.

El estudio CARE, utilizando la pravastatina, ha demostrado que estos efectos tienen lugar tanto en personas con niveles elevados de LDL como en individuos con niveles de LDL normales aunque este estudio incluye sólo a personas con niveles «normales» de colesterol: $<240 \mathrm{mg} / \mathrm{dl}$.

Tabla 3

Cifras de tensión arterial en relación con el Tratamiento

\begin{tabular}{|ccc|}
\hline Valores T.A & Frecuencia & Tratamiento \\
\hline TAS $>140$ & $43.8 \%$ & $77 \%$ \\
TAD $>90$ & $11.5 \%$ & $78.1 \%$ \\
\hline
\end{tabular}

Tabla 4

Comparación de datos en prevención secundaria

\begin{tabular}{|lllccccc|}
\hline & $\begin{array}{c}\text { LDLc } \\
\text { (media) }\end{array}$ & $\begin{array}{c}\text { COLt } \\
\text { (media) }\end{array}$ & $\begin{array}{c}\text { EDAD } \\
\text { (media) }\end{array}$ & $\begin{array}{c}\text { HIPOLIPEMIANTE } \\
(\text { LDL }>130)\end{array}$ & IMC & SEXO \\
\hline DRECE & 186 & 287 & - & $52,19 \%$ & - & & $M$ \\
PREVESE & 149,72 & 210 & 65,2 & $33 \%$ & $59 \%$ & $\mathbf{7 8 , 3 \%}$ & $21,7 \%$ \\
PRECIAR & 149,7 & 221,78 & 72,4 & $43,6 \%$ & $6 \%$ & $\mathbf{5 7 , 2 \%}$ & $42,8 \%$ \\
\hline
\end{tabular}


En los resultados de algunos estudios se pueden observar que los beneficios obtenidos con tratamiento quirúrgico son equivalentes a los obtenidos con tratamiento médico con inclusión de estatinas, aunque el estudio AVERT no demostró, de forma significativamente estadística, que la terapia con dosis elevadas de atorvastatina redujese el riesgo coronario en comparación con la realización de angioplastia.

Comparando los datos, en nuestro estudio aparecen cifras medias de colesterol total de 221,78 y de LDL de 149,7, teniendo en cuenta que se trata de prevención secundaria en personas con cardiopatía isquémica ya existente; el estudio $C A R E$ (aunque es un estudio intervencionista y no epidemiológico) revela unas cifras medias de 209 para el COL-T y de 139 para LDL. En el estudio PREVESE 98 se registran cifras de 210 de colesterol total y de 149,72 de LDL, como media. Tomando como hipercolesterolemia cifras de LDL por encima de 130, nuestro estudio refleja que tan sólo un 43,4 \% reciben tratamiento farmacológico y además, el $96 \%$ de los pacientes que reciben dicho tratamiento no alcanzan el control óptimo establecido por el NCEP-II y otras sociedades; frente al $33 \%$ que señala el estudio PREVESE. Llama poderosamente la atención el elevado porcentaje de casos no tratados, pero más llamativo todavía es comprobar en nuestro estudio que tan sólo un $24,2 \%$ de los pacientes tratados se mantenía con niveles de LDL por debajo de 130.

Respecto al registro de otros factores de riesgo, la somatometría aparece contemplada en el $76 \%$ de los casos, frente al $59 \%$ del estudio PREVESE.

Los resultados de nuestro estudio revelan que el diagnóstico de las dislipemias, así como el control del resto de factores de riesgo cardiovascular en prevención secundaria, no se han adecuado en gran medida a los criterios de calidad de este proceso asistencial y señalan, en consecuencia, la existencia de un amplio margen de mejora. Es muy llama- tivo el dato de que tan sólo un 60,8\% de todos los casos tenga registrado un perfil lipídico completo en el último año y de éstos sólo el 43,6\% de los que reflejaban un nivel LDL $>130$ llevaban tratamiento hipolipemiante ${ }^{6-8}$. Asimismo, la amplia variabilidad en el cumplimiento de los criterios entre los diferentes casos estudiados, sugiere una ausencia de pautas de actuación concretas y normalizadas en el nivel de atención primaria del sistema de salud y señala la existencia de un problema de calidad, de dimensiones importantes en el control de las dislipemias y del resto de factores de riesgo cardiovascular $^{9,12}$.

Es de destacar el hecho de que todos los factores estructurales analizados en el estudio están asociados en alguna medida con la calidad de la atención en el control de los factores de riesgo cardiovascular en cuanto a prevención secundaria. Sin embargo, los factores estructurales son necesarios pero no siempre son suficientes para garantizar una atención sanitaria correcta.

El registro de factores de riesgo ha sido el criterio de calidad con mayor proporción de cumplimientos, sin embargo su utilización explícita ha sido ínfima para valorar el riesgo cardiovascular, lo que facilitaría un manejo terapéutico más adecuado.

En resumen, la calidad del registro (proceso) ha sido mucho mayor que la calidad de resultados, un planteamiento que nos lleva a cuestionarnos si realmente estamos incidiendo en disminuir los factores de riesgo en la prevención secundaria cardiovascular. En cualquier caso, es evidente la existencia de un amplio margen de mejora y una distribución heterogénea en la calidad de la atención. El seguimiento de los factores de riesgo en estos pacientes es claramente un servicio a mejorar.

Pueden hacerse algunas consideraciones acerca de lo que significaría, en cuanto a beneficios cardiovasculares, alcanzar los objetivos terapéuticos previamente consensua- 
dos: Deberíamos tratar a todos las personas que puedan considerarse para prevención secundaria, y además con dosis de fármacos lo suficientemente eficaces. Este hecho supondría que el número de individuos en tratamiento hipolipemiante sería considerable y el costo sanitario importante. Sin embargo los análisis coste-beneficio sobre el tratamiento hipolipemiante en prevención secundaria han dado resultados favorables para el uso de estatinas en estas circunstancias. De hecho, los análisis del estudio $4 \mathrm{~S}$ demuestran que más del $88 \%$ del costo del fármaco se recupera con el ahorro en intervenciones sanitarias conseguido (independientemente de los años de vida ganados $)^{6,8,14}$.

\section{BIBLIOGRAFÍA}

1. The long term intervention with pravastatin in ischaemic disease (LIPID) study group. Prevention of cardiovascular events and death with coronary heart disease and a broad range initial cholesterol levels. NJM 1998; 339 (19). 1349-1357.

2. Postinfarto de miocardio. Las decisiones terapéuticas al alta hospitalaria en España (estudio PREVESE). Rev Clín Esp 1996; monográfico 4: 196.

3. J.A. Gómez Gerique et al. Situación actual del tratamiento hipolipemiante en España. Clin Invest Arterioesclerosis 1997; 9(2):

4. Frank M Sacks et al. Características basales del estudio CARE (Colesterol y acontecimientos recurrentes) en la prevención secundaria de pacientes con concentraciones séricas medias de colesterol.

5. Efecto de simvastatina en los síntomas y signos isquémicos en el Scandinavian Simvastatin Survival Study (4S). Am J Cardiol 1998; Feb. 1; 81.
6. Cost-effectiveness of statins. Am J Cardiol 1998 Dec 1; 82 (11): 1357-63.

7. Improving health outcomes without increasing costs: maximizing the full potential of lipid reduction therapy in the primary and secondary prevention of coronary heart disease. Curr Opin Lipidol 1997; 8: 369-74.

8. Cost-effectiveness of primary and secondary prevention in cardiovascular diseases. Eur Heart J 1998; 19 Suppl C: C59-65.

9. Primary and secondary prevention of coronary heart disease: what can we afford? Z Kardiol 1999; 88: 85-9.

10. Lipid-lowering for prevention of coronary heart disease. Clin Sci (Colch). 1996; 91: 399-413.

11. Statins in secondary prevention of coronary heart disease. Wien Med Wochenschr 1999; 149: 125-8

12. Evidence-based medicine in cardiology with an example of secondary prevention of coronary heart disease. Gohlke H. Z Arztl Fortbiuld Qualitatssich 1999; 93: 403-7.

13. Capewell $\mathrm{S}$ et al. Increasing the impact of cardiological treatments. How best to reduce deaths. Eur Heart J 1999; 20: 1386-92.

14. Lipid management in patients at moderate risk for coronary heart disease: insights from the Air Force/Texas Coronary Atherosclerosis Prevention Study. Gotto AM JAm J Med 1999; 107: 36S-39S.

15. The sixth report of the Joint National Committee on prevention, detection, evaluation and treatment og high blood pressure. Arch Intern Med 1997; 157: 2413-2446.

16. National Cholesterol Education Program. Second report. ATP Panel II. Jama 1993; 23: 269. 